\title{
Small Solutions of Quadratic Congruences, and Character Sums with Binary Quadratic Forms
}

\author{
D.R. Heath-Brown \\ Mathematical Institute, Oxford
}

\section{Introduction}

Let $Q(\mathbf{x})=Q\left(x_{1}, \ldots, x_{n}\right) \in \mathbb{Z}\left[x_{1}, \ldots, x_{n}\right]$ be a quadratic form. This paper, which may be seen as a continuation of the author's earlier work [10], [11] seeks to understand the smallest solution of the congruence $Q(\mathbf{x}) \equiv 0(\bmod q)$ in non-zero integers $\mathbf{x}$. Thus we shall set

$$
m(Q ; q):=\min \left\{\|\mathbf{x}\|: \mathbf{x} \in \mathbb{Z}^{n}-\{\mathbf{0}\}, Q(\mathbf{x}) \equiv 0(\bmod q)\right\}
$$

where $\|\mathbf{x}\|$ denotes the Euclidean norm, and ask (in the first instance) about

$$
B_{n}(q):=\max _{Q} m(Q ; q),
$$

where the maximum is taken over all integral quadratic forms in $n$ variables. (This definition differs slightly from that used in [10] and [11.) The interested reader may refer to Baker [1, Chapter 9] for an account of this problem and its applications.

It is trivial that $B_{n}(q)$ is non-increasing as a function of $n$. When $q$ is square-free it is easy to see that $B_{n}(q) \geq q$ for $n=1$ or 2 . Moreover the form $Q(\mathbf{x})=x_{1}^{2}+\ldots+x_{n}^{2}$ has $m(Q ; q) \geq q^{1 / 2}$ so that $B_{n}(q) \geq q^{1 / 2}$ for every $q$ and every $n$. When $n=3$ and $q$ is square-free one has

$$
B_{3}(q) \geq m(Q ; q) \geq q^{2 / 3}+O\left(q^{1 / 3}\right)
$$

for a suitable singular form

$$
Q\left(x_{1}, x_{2}, x_{3}\right)=\left(x_{1}-b x_{2}\right)^{2}-a\left(x_{2}-b x_{3}\right)^{2} .
$$

(Details for the case in which $q$ is prime are given in [10, Theorem 3] but the argument readily extends to any square-free $q$.) It is reasonable to conjecture that such lower bounds represent the true order of magnitude for $B_{n}(q)$ in general, so that one would have

$$
B_{n}(q) \ll_{\varepsilon} \begin{cases}q^{2 / 3+\varepsilon}, & n=3 \\ q^{1 / 2+\varepsilon}, & n \geq 4\end{cases}
$$

for any fixed $\varepsilon>0$ (uniformly in $n$, by the non-increasing property).

A basic upper bound for $B_{n}(q)$ was provided by Schinzel, Schlickewei and Schmidt [16, who showed that

$$
B_{n}(q) \ll\left\{\begin{array}{cc}
q^{1 / 2+1 /(2 n)}, & n \text { odd }, \\
q^{1 / 2+1 /(2 n-2)}, & n \text { even. }
\end{array}\right.
$$


In particular one sees that $q^{2 / 3}$ is the true order of magnitude of $B_{3}(q)$, at least when $q$ is square-free.

For $n \geq 4$ and any $\varepsilon>0$ one has

$$
B_{n}(q) \ll_{\varepsilon} q^{1 / 2+\varepsilon}
$$

if $q$ has at most 2 prime factors, (see the author [11, Theorem 1]); that

$$
B_{4}(q) \ll_{\varepsilon} q^{5 / 8+\varepsilon}
$$

(see [11, Theorem 2]); and that

$$
B_{n}(q) \ll_{\varepsilon, n} q^{1 / 2+3 / n^{2}+\varepsilon}
$$

for every even $n \geq 2$ (see [11, Theorem 3]). Indeed a number of other such bounds are possible.

It might appear from the above discussion that our question is completely resolved for $n=3$, but wide open for $n \geq 4$. None the less, the main goal of this paper is a further exploration of the situation for $n=3$ (!) It will be observed that the example (1.1) is a singular form. It turns out that one can do better if one restricts attention to ternary forms which are nonsingular modulo $q$. Before stating our result we should make two simple observations. Firstly, if $q=q_{0}^{2} q_{1}$ and $q_{1} \mid Q(\mathbf{x})$, then $q \mid Q\left(q_{0} \mathbf{x}\right)$. It follows that $m(Q ; q) \leq q_{0} m\left(Q ; q_{1}\right)$. In particular, if we have proved that $B_{n}(q) \ll q^{\theta}$ for all square-free $q$, for some exponent $\theta \geq \frac{1}{2}$, then we may deduce that $B_{n}(q) \ll q^{\theta}$ for every $q$. Secondly, if $q=2 q_{1}$ and $q_{1} \mid Q(\mathbf{x})$, then $q \mid Q(2 \mathbf{x})$. It follows in this case that $m(Q ; q) \leq 2 m\left(Q ; q_{1}\right)$. Once again, if we have proved that $B_{n}(q) \ll q^{\theta}$ for all odd square-free $q$, for some exponent $\theta$, then we may deduce that $B_{n}(q) \ll q^{\theta}$ for every square-free $q$. These observations allow us to focus on odd square-free $q$. Indeed we shall assume without further comment throughout the remainder of this paper that $q$ is odd and square-free. In this situation we can represent $Q(\mathbf{x})$ modulo $q$ via a symmetric integer matrix, which we also denote by $Q$, by abuse of notation.

We now define

$$
B_{3}^{*}(q):=\max _{Q} m(Q ; q),
$$

where the maximum is taken over all integral ternary quadratic forms $Q$ with $(\operatorname{det}(Q), q)=1$. This notation allows us to state our principal result.

Theorem 1 Let $q \in \mathbb{N}$ be odd and square-free, and let $\varepsilon>0$ be given. Then

$$
B_{3}^{*}(q) \ll_{\varepsilon} q^{5 / 8+\varepsilon} .
$$

So we see that we can go below the exponent $2 / 3$ which is the limiting exponent for $B_{3}(q)$. We now have the same exponent $5 / 8$ for (non-singular) forms in 3 variables as we previously had for 4 variables. (However it is explained in [11] that one can reduce the exponent to $13 / 21$ with more work, in the 4 variable case.)

It now seems that one should conjecture a bound

$$
B_{3}^{*}(q) \ll_{\varepsilon} q^{1 / 2+\varepsilon} .
$$


The proof of Theorem 1 proceeds by reducing the problem to a second question, which we now explain. If $Q$ is a quadratic form in $n \geq 2$ variables we write

$$
\widehat{m}(Q ; q):=\min \left\{\|\mathbf{x}\|: \mathbf{x} \in \mathbb{Z}^{n}-\{\mathbf{0}\}, \exists t \in \mathbb{Z}, Q(\mathbf{x}) \equiv t^{2}(\bmod q)\right\}
$$

and

$$
\widehat{B}_{n}(q):=\max _{Q} \widehat{m}(Q ; q),
$$

where the maximum is taken over all integral quadratic forms in $n$ variables such that $(\operatorname{det}(Q), q)=1$.

We then have the following result.

Lemma 1 Let $q \in \mathbb{N}$ be odd and square-free. Then if $Q$ is a ternary quadratic form with $(\operatorname{det}(Q), q)=1$ we have

$$
m(Q ; q) \ll q^{1 / 2} \widehat{m}\left(-Q^{\operatorname{adj}} ; q\right)^{1 / 2}
$$

where $Q^{\text {adj }}$ is the adjoint matrix for $Q$. In particular one has

$$
B_{3}^{*}(q) \ll q^{1 / 2} \widehat{B}_{3}(q)^{1 / 2} .
$$

This naturally leads us to speculate about the size of $\widehat{B}_{3}(q)$, and the natural conjecture is that

$$
\widehat{B}_{3}(q) \ll_{\varepsilon} q^{\varepsilon}
$$

for any fixed $\varepsilon>0$. Of course Lemma 1 immediately shows that this latter conjecture implies (1.2).

If $q$ is odd and square-free there is a real character

$$
\chi_{d}(m)=\left(\frac{m}{d}\right)
$$

for each divisor $d$ of $m$, and the congruence $Q(\mathbf{x}) \equiv t^{2}(\bmod q)$ will have a solution $t$ if and only if

$$
\sum_{d \mid q} \chi_{d}(Q(\mathbf{x}))>0
$$

We can therefore attempt to show that $\widehat{B}_{3}(q)$ is small by investigating the character sums

$$
S(\chi, B, Q):=\sum_{\|\mathbf{x}\| \leq B} \chi(Q(\mathbf{x}))
$$

for primitive characters $\chi$ to modulus $d>1$. If we can show that

$$
S(\chi, B, Q) \ll B^{3-\delta}
$$

for some fixed $\delta>0$, for every primitive $\chi$ to modulus $d \geq 2$, then we will be able to deduce that $\widehat{m}_{3}(Q ; q) \leq B$, since one has $S(1, B, Q) \gg B^{3}$ for the trivial character.

It seems plausible that (1.4) should hold for $B \geq q^{\eta}$, for any fixed $\eta>0$, and with $\delta=\delta(\eta)>0$. This would suffice for (1.3), and hence also for (1.2). 
One standard procedure to estimate sums such as $S(\chi, B, Q)$ is to complete the sum and use bounds of Weil-Deligne type. It is very instructive to carry this out in detail. What one finds, if $d=q$ for example, is essentially that

$$
S(\chi, B, Q) \ll \frac{B^{3}}{q^{3}} \sum_{\underline{\mathrm{y}} \in \mathbb{Z}^{3}} W\left(\frac{q}{B} \underline{\mathrm{y}}\right) S(\underline{\mathrm{y}}),
$$

where $W \ll 1$ is a suitable weight function and

$$
S(\underline{\mathrm{y}}):=\sum_{\mathbf{x}(\bmod q)} e_{q}(\underline{\mathrm{y}} \cdot \mathbf{x}) \chi(Q(\mathbf{x})) .
$$

These complete sums can be computed explicitly. Taking $q$ to be prime for simplicity, and assuming that $q \nmid \operatorname{det}(Q)$, one finds that $S(\mathrm{y})$ is of order $q^{2}$ when $q \mid Q^{\operatorname{adj}}(\underline{y})$, and of order $q$ otherwise. This may be something of a surprise, since one typically expects complete sums in $n$ variables to have size around $q^{n / 2}$. As a result this analysis leads to a bound which one may think of as

$$
S(\chi, B, Q) \ll_{\varepsilon} q^{\varepsilon}\left(q+B^{3} q^{-1} \#\left\{\underline{\mathrm{y}} \ll q / B: q \mid Q^{\operatorname{adj}}(\underline{\mathrm{y}})\right\}\right) .
$$

Since we have estimated $m(Q ; q)$ in terms of sums $S\left(\chi, B, Q^{\text {adj }}\right)$ it is apparent that the above analysis ultimately connects small solutions of $q \mid Q(\mathbf{x})$ with small solutions of $q \mid Q(\underline{\mathrm{y}})$. In fact the argument is not completely circular, and one can show in this way that $B_{3}^{*}(q) \ll_{\varepsilon} q^{2 / 3+\varepsilon}$, at least when $q$ is prime. Alternatively one can provide an upper bound for

$$
\#\left\{\underline{\mathrm{y}} \ll q / B: q \mid Q^{\mathrm{adj}}(\underline{\mathrm{y}}), \underline{\mathrm{y}} \text { primitive }\right\}
$$

by using $O\left((q / B)^{3 / 2}\right)$ plane slices of the type $\underline{a} \cdot \underline{y}=0$. Each such slice produces a binary quadratic form of rank 1 or 2 , which will have $O(1)$ primitive zeros modulo $q$, under the assumption that $q$ is prime. In this way one finds that

$$
\#\left\{\underline{\mathrm{y}} \ll q / B: q \mid Q^{\mathrm{adj}}(\underline{\mathrm{y}})\right\} \ll(q / B)^{3 / 2},
$$

and hence

$$
S(\chi, B, Q) \ll_{\varepsilon} q^{\varepsilon}\left(q+B^{3 / 2} q^{1 / 2}\right) .
$$

We therefore have a non-trivial bound for $B \geq q^{1 / 3+\delta}$. Unfortunately this merely yields $\widehat{B}_{3}(q) \ll_{\varepsilon} q^{1 / 3+\varepsilon}$ and hence $B_{3}^{*}(q) \ll q^{2 / 3+\varepsilon}$.

We have been unable to obtain a non-trivial bound for $S(\chi, B, Q)$ when $B \leq q^{1 / 3}$. However, if one replaces $Q$ by a binary form one can do better. Indeed the following result of Chang [7, Theorem 11] is the main inspiration for this paper.

Theorem 2 (Chang). For any $\varepsilon>0$ there is a corresponding $\delta>0$ such that

$$
\left|\sum_{X^{\prime}<x \leq X+X^{\prime} Y^{\prime}<y \leq Y+Y^{\prime}} \sum \chi\left(x^{2}+a x y+b y^{2}\right)\right|<p^{-\delta} X Y
$$

for any non-trivial character $\chi$ modulo $p$, any $X, Y>p^{1 / 4+\varepsilon}$, and any integers $a, b$ with $a^{2} \not \equiv 4 b(\bmod p)$. 
This improves on the corresponding results of Burgess [5] and [6], which were non-trivial only for $X, Y>p^{1 / 3+\varepsilon}$.

The proof of Chang's result crucially uses the fact that a binary quadratic form over $\mathbb{F}_{p}$ factors over $\mathbb{F}_{p^{2}}$, and of course this limits the approach to the case $n=2$. Since we are interested in composite $q$ we will require a variant of Theorem 2, The argument in [7] splits into two rather different cases, one in which the form factors over $\mathbb{F}_{p}$, and one in which it does not. In order to handle composite $q$ we need to devise a treatment which handles both cases in the same way. Our result is the following.

Theorem 3 Let $\varepsilon>0$ and an integer $r \geq 3$ be given, and suppose that $C \subset \mathbb{R}^{2}$ is a convex set contained in a disc $\left\{\mathbf{x} \in \mathbb{R}^{2}:\left\|\mathbf{x}-\mathbf{x}_{0}\right\| \leq R\right\}$. Let $q \geq 2$ be odd and square-free, and let $\chi$ be a primitive character to modulus $q$. Then if $Q(x, y)$ is a binary quadratic form with $(\operatorname{det}(Q), q)=1$ we have

$$
\sum_{(x, y) \in C} \chi(Q(x, y)) \ll_{\varepsilon, r} R^{2-1 / r} q^{(r+2) /\left(4 r^{2}\right)+\varepsilon}
$$

for $q^{1 / 4+1 / 2 r} \leq R \leq q^{5 / 12+1 / 2 r}$.

For comparison we observe that the standard Burgess bound [4, Theorem 2] yields

$$
\sum_{x, y \leq R} \chi(x y) \ll_{\varepsilon, r} R^{2-2 / r} q^{(r+1) /\left(2 r^{2}\right)+\varepsilon},
$$

relative to which our theorem has a loss of $\left(R q^{-1 / 4}\right)^{1 / r}$. In Section 3 we will apply Theorem [3 with $C=\left\{\mathbf{x} \in \mathbb{R}^{2}:\|\mathbf{x}\| \leq R\right\}$ and $R=q^{1 / 4+\delta}$. Taking $r>(2 \delta)^{-1}$ we will be able to deduce that $\widehat{B}_{2}(q) \ll_{\varepsilon} q^{1 / 4+\delta}$. We then go on to conclude that $\widehat{B}_{3}(q) \ll_{\varepsilon} q^{1 / 4+\varepsilon}$ and hence, via Lemma 1, that $B_{3}^{*}(q) \ll q^{5 / 8+\varepsilon}$.

Before embarking on the proofs we need to mention one point of notation. We shall follow the common convention that the small positive number $\varepsilon$ will be allowed to change between appearances, allowing us to write $q^{\varepsilon} \log q \ll_{\varepsilon} q^{\varepsilon}$, for example.

\section{Proof of Lemma 1}

Suppose that $-Q^{\text {adj }}(\mathbf{a}) \equiv t^{2}(\bmod q)$ with $\|\mathbf{a}\|=\widehat{m}\left(-Q^{\text {adj }} ; q\right)$ and $\mathbf{a} \neq \mathbf{0}$. Write $\mathbf{a}=\alpha \mathbf{a}_{0}$ with $\alpha \in \mathbb{N}$ and $\mathbf{a}_{0}$ primitive, and let

$$
\Lambda:=\left\{\mathbf{x} \in \mathbb{Z}^{3}: \mathbf{a}_{0} \cdot \mathbf{x}=0\right\}
$$

This will be a 2-dimensional lattice of determinant $\left\|\mathbf{a}_{0}\right\|$. Let $\mathbf{x}_{1}$ be the shortest non-zero vector in $\Lambda$, and $\mathbf{x}_{2}$ the shortest vector non-proportional to $\mathbf{x}_{1}$. Then $\mathbf{x}_{1}$ and $\mathbf{x}_{2}$ form a basis for $\Lambda$, and we have

$$
\left\|\mathbf{x}_{1}\right\| \cdot\left\|\mathbf{x}_{2}\right\| \ll\left\|\mathbf{a}_{0}\right\|
$$

and

$$
\mathbf{x}_{1} \wedge \mathbf{x}_{2}= \pm \mathbf{a}_{0}
$$


We proceed to write $R(u, v):=Q\left(u \mathbf{x}_{1}+v \mathbf{x}_{2}\right)$, so that $R$ is a binary quadratic form. We then have

$$
\operatorname{det}(R)=\operatorname{det}\left(Q\left(u \mathbf{x}_{1}+v \mathbf{x}_{2}\right)\right)=Q^{\text {adj }}\left(\mathbf{x}_{1} \wedge \mathbf{x}_{2}\right)=Q^{\text {adj }}\left(\mathbf{a}_{0}\right)
$$

as an identity, so that

$$
-\alpha^{2} \operatorname{det}(R)=-Q^{\operatorname{adj}}(\mathbf{a}) \equiv t^{2}(\bmod q) .
$$

Let $(q, \alpha)=q_{0}$ and $q=q_{0} q_{1}$. It follows that $R$ factors over $\mathbb{F}_{p}$ for every prime factor $p$ of $q_{1}$. We may then use the Chinese Remainder Theorem to write $R(u, v) \equiv L_{1}(u, v) L_{2}(u, v)\left(\bmod q_{1}\right)$ for certain integral linear forms $L_{1}$ and $L_{2}$.

Our strategy is now to find a short vector $\mathbf{x} \in \Lambda$ such that $q_{1} \mid L_{1}(u, v)$. We will then automatically have $q_{1} \mid R(u, v)$ and hence $q_{1} \mid Q\left(u \mathbf{x}_{1}+v \mathbf{x}_{2}\right)$. This will produce $q \mid Q(\mathbf{x})$ with $\mathbf{x}=q_{0}\left(u \mathbf{x}_{1}+v \mathbf{x}_{2}\right)$.

Let

$$
U:=\left(q_{1} \frac{\left\|\mathbf{x}_{2}\right\|}{\left\|\mathbf{x}_{1}\right\|}\right)^{1 / 2} \text { and } V:=\left(q_{1} \frac{\left\|\mathbf{x}_{1}\right\|}{\left\|\mathbf{x}_{2}\right\|}\right)^{1 / 2},
$$

so that $U V=q_{1}$. Then an easy application of the pigeon-hole principle shows that one can find $(u, v) \in \mathbb{Z}^{2}-\{(0,0)\}$ with $q_{1} \mid L_{1}(u, v)$ and satisfying $|u| \leq U$ and $|v| \leq V$. We then deduce that

$$
\begin{aligned}
\|\mathbf{x}\| & =q_{0}\left\|u \mathbf{x}_{1}+v \mathbf{x}_{2}\right\| \\
& \leq q_{0}\left(U\left\|\mathbf{x}_{1}\right\|+V\left\|\mathbf{x}_{2}\right\|\right) \\
& =2 q_{0}\left(q_{1}\left\|\mathbf{x}_{1}\right\| \cdot\left\|\mathbf{x}_{2}\right\|\right)^{1 / 2} \\
& \ll q_{0}\left(q_{1}\left\|\mathbf{a}_{0}\right\|\right)^{1 / 2} \\
& =q^{1 / 2}\left(q_{0}\left\|\mathbf{a}_{0}\right\|\right)^{1 / 2} \\
& \leq q^{1 / 2}\left(\alpha\left\|\mathbf{a}_{0}\right\|\right)^{1 / 2} \\
& =q^{1 / 2}\|\mathbf{a}\|^{1 / 2}
\end{aligned}
$$

by (2.1). Since $\mathbf{x}$ must be non-zero we deduce that

$$
m(Q ; q) \ll(q\|\mathbf{a}\|)^{1 / 2}=q^{1 / 2} \widehat{m}\left(-Q^{\text {adj }} ; q\right)^{1 / 2},
$$

as required.

\section{Deduction of Theorem 1}

In this section we will show how Theorem 1 follows from Theorem 3 . Clearly it suffices to prove that $\widehat{m}(Q ; q) \ll_{\varepsilon} q^{1 / 4+\varepsilon}$ uniformly for any ternary form $Q$ with $(\operatorname{det}(Q), q)=1$.

Our first task is to establish the following corollary to Theorem 3 ,

Lemma 2 For any $\delta>0$ there is a corresponding $\eta>0$ such that, if $q>1$ and $C \subset \mathbb{R}^{2}$ is a convex set contained in a disc $\left\{\mathbf{x} \in \mathbb{R}^{2}:\left\|\mathbf{x}-\mathbf{x}_{0}\right\| \leq R\right\}$, then

$$
\sum_{(x, y) \in C} \chi(Q(x, y)) \ll_{\delta} R^{2-\eta}
$$

for $R \geq q^{1 / 4+\delta}$, uniformly for every primitive character $\chi$ modulo $q$ and for every binary quadratic form $Q$ with $(\operatorname{det}(Q), q)=1$. 
We prove this in three steps, beginning with the case in which $q^{1 / 4+\delta} \leq R \leq$ $q^{5 / 12}$. In this range we choose

$$
r=3+[1 / \delta], \quad \eta=1 /\left(r^{2}+4 r\right) \text { and } \varepsilon=\eta / 4 .
$$

Then $r \geq 3$ and $1 / 4+\delta \geq 1 / 4+1 / r$, so that

$$
q \leq R^{1 /(1 / 4+\delta)} \leq R^{4 r /(r+4)} .
$$

Thus Theorem 3 produces

$$
\begin{aligned}
\sum_{(x, y) \in C} \chi(Q(x, y)) & \ll_{\varepsilon, r} \quad R^{2-1 / r} q^{(r+2) /\left(4 r^{2}\right)+\varepsilon} \\
& \ll_{\delta} R^{2-1 / r+(r+2) / r(r+4)+4 \varepsilon} \\
& =R^{2-2 / r(r+4)+\eta} \\
& =R^{2-\eta} .
\end{aligned}
$$

Next, when $q^{5 / 12} \leq R \ll q$, we cover $\mathbb{R}^{2}$ with disjoint squares of side $q^{5 / 12}$ to obtain a partition of $C$ into $O\left(R^{2} q^{-5 / 6}\right)$ convex subsets, each with diameter at most $q^{5 / 12}$. On applying the result above with $\delta=1 / 6$ we find that

$$
\sum_{(x, y) \in C} \chi(Q(x, y)) \ll R^{2} q^{-5 / 6}\left(q^{5 / 12}\right)^{2-\eta} \ll R^{2-5 \eta / 12}
$$

for some absolute constant $\eta>0$.

Finally we examine the case $R \gg q$. This time we cover $C$ with squares of side $q$, and observe that

$$
\sum_{x, y(\bmod q)} \chi(Q(x, y))=0
$$

(By multiplicativity it suffices to prove this when $q$ is prime, in which case it is an easy exercise, relying on the fact that $Q$ is nonsingular modulo $q$.) Since $C$ will be partitioned into $O\left(R^{2} q^{-2}\right)$ complete squares and $O\left(R q^{-1}\right)$ partial squares we may use the result (3.1) to conclude that

$$
\sum_{(x, y) \in C} \chi(Q(x, y)) \ll R q^{-1} q^{2-5 \eta / 12} \leq R^{2-5 \eta / 12},
$$

and the lemma follows.

We next estimate $\widehat{m}(Q ; q)$ for binary forms $Q$.

Lemma 3 For any fixed $\delta>0$ we have

$$
\widehat{m}(Q ; q) \ll_{\delta} q^{1 / 4+\delta}
$$

uniformly over odd square-free moduli $q$, and over binary forms $Q$ subject to $(\operatorname{det}(Q), q)=1$.

As already noted in the introduction, if we let $d$ run over all divisors of $q$ then if $\sum_{d} \chi_{d}(m)>0$ we must have $m \equiv t^{2}(\bmod q)$ for some integer $t$. It follows that $\widehat{m}(Q ; q) \leq R$ provided that

$$
\sum_{d \mid q} \sum_{\|(x, y)\| \leq R} \chi_{d}(Q(x, y))>0 .
$$


The number of divisors of $q$ is $O_{\varepsilon}\left(q^{\varepsilon}\right)$, for any $\varepsilon>0$. Choosing $\varepsilon=\eta / 8$ it follows from Lemma 2 that if $R \geq q^{1 / 4+\delta}$ then

$$
\sum_{d \mid q, d>1} \sum_{\|(x, y)\| \leq R} \chi_{d}(Q(x, y)) \ll_{\delta} R^{2-\eta} q^{\varepsilon} \ll R^{2-\eta / 2} .
$$

On the other hand

$$
\sum_{\|(x, y)\| \leq R} 1 \gg R^{2}
$$

and Lemma 3 follows.

Finally we need to estimate $\widehat{B}_{3}(q)$ in terms of $\widehat{B}_{2}(q)$.

Lemma 4 We have

$$
\widehat{B}_{3}(q) \ll_{\varepsilon} q^{\varepsilon} \widehat{B}_{2}(q)
$$

for any fixed $\varepsilon>0$.

Once this is proved we may deduce from Lemma 3 that $\widehat{B}_{3}(q) \ll_{\varepsilon} q^{1 / 4+\varepsilon}$ for any $\varepsilon>0$, whence Lemma 1 yields $B_{3}^{*}(q) \ll_{\varepsilon} q^{5 / 8+\varepsilon}$. This is the result required for Theorem 1 .

To establish Lemma 4 we will find short vectors

$$
\left(a_{1}, a_{2}\right),\left(a_{3}, a_{4}\right),\left(a_{5}, a_{6}\right) \in \mathbb{Z}^{2}
$$

such that the form

$$
R(u, v):=Q\left(a_{1} u+a_{2} v, a_{3} u+a_{4} v, a_{5} u+a_{6} v\right)
$$

has $(\operatorname{det}(R), q)=1$. We can then choose $u, v \ll \widehat{B}_{2}(q)$, not both zero, such that $R(u, v)$ is a square modulo $q$, which will produce a corresponding vector

$$
\mathbf{x}=\left(a_{1} u+a_{2} v, a_{3} u+a_{4} v, a_{5} u+a_{6} v\right)
$$

for which $Q(\mathbf{x})$ is a square modulo $q$, and with

$$
\|\mathbf{x}\| \ll\|(u, v)\| \max \left(a_{1}, \ldots, a_{6}\right) .
$$

If $\mathbf{x}$ were to vanish the three vectors (3.2) would all have to be proportional. But then the form (3.3) would have rank at most 1 , so that $\operatorname{det}(R)=0$. This would contradict our assumption that $(\operatorname{det}(R), q)=1$. It follows that we must have $\mathbf{x} \neq \mathbf{0}$. Thus to complete the proof of Lemma 4 it will suffice to show that we can choose the coefficients $a_{1}, \ldots, a_{6}$ to be of size $O_{\varepsilon}\left(q^{\varepsilon}\right)$.

Define

$$
\Delta\left(a_{1}, \ldots, a_{6}\right):=\operatorname{det}\left(Q\left(a_{1} u+a_{2} v, a_{3} u+a_{4} v, a_{5} u+a_{6} v\right)\right) .
$$

This will be a sextic form in the 6 variables $a_{1}, \ldots, a_{6}$. We claim that for each prime factor $p$ of $q$ there is at least one choice of $\mathbf{a} \in \mathbb{Z}^{6}$ such that $p \nmid \Delta(\mathbf{a})$. Since we can diagonalize $Q$ by a unimodular transformation over $\mathbb{F}_{p}$ a moment's reflection shows that it is enough to verify the claim when $Q$ is a diagonal form. However the result is trivial in this case since $p \nmid \operatorname{det}(Q)$.

We can now call on the following lemma, which we will prove in a moment. 
Lemma 5 Let $\varepsilon, \delta>0$ be given. Suppose that $F\left(x_{1}, \ldots, x_{n}\right) \in \mathbb{Z}\left[x_{1}, \ldots, x_{n}\right]$ is a form of degree $d$, and let $q \in \mathbb{N}$. Assume that for every prime divisor $p$ of $q$ there is at least one $\mathbf{a} \in \mathbb{Z}^{n}$ such that $p \nmid F(\mathbf{a})$. Then

$$
\#\left\{\mathbf{a} \in \mathbb{N}^{n}: \max a_{i} \leq A,(F(\mathbf{a}), q)=1\right\} \gg_{d, n, \varepsilon} A^{n} q^{-\varepsilon}
$$

as soon as $A \geq q^{\delta}$ and $q \gg_{n, d} 1$.

This result shows that we have at least one vector a of size $\|\mathbf{a}\| \ll q^{\varepsilon}$ such that $\Delta(\mathbf{a})$ is coprime to $q$, which suffices to complete the proof.

It remains to prove Lemma 5, Define

$$
N(e):=\#\{\mathbf{a}(\bmod e): e \mid F(\mathbf{a})\}
$$

for each $e \in \mathbb{N}$. Then $N(e)$ is multiplicative, and $N(p)<p^{n}$ for $p \mid q$ by the hypothesis of the lemma. Moreover, when $N(p)<p^{n}$ the form $F$ cannot vanish identically modulo $p$, whence $N(p) \ll_{d, n} p^{n-1}$. It follows that

$$
N(e) \ll_{d, n, \eta} e^{n-1+\eta}
$$

for $e \mid q$, for any fixed $\eta>0$.

We now consider

$$
N(e, A):=\#\left\{\mathbf{a} \in \mathbb{N}^{n}: \max a_{i} \leq A, e \mid F(\mathbf{a})\right\} .
$$

The set $(0, A]^{n}$ contains $[A / e]^{n}$ disjoint cubes of side-length $e$, and is included in a union of $(1+[A / e])^{n}$ such cubes. It follows that

$$
N(e, A)=\frac{A^{n}}{e^{n}} N(e)+O_{n}\left(A^{n-1} e^{1-n} N(e)\right)=\frac{A^{n}}{e^{n}} N(e)+O_{d, n, \eta}\left(A^{n-1} e^{\eta}\right)
$$

when $e \mid q$ and $e \leq A$. To handle larger values of $e$ we use a rather general result of Browning and Heath-Brown [2]. For each $p_{i} \mid q$ let $V_{i}$ be the affine variety over $\mathbb{F}_{p_{i}}$ given by $F=0$. Since $F$ does not vanish identically modulo $p_{i}$ this has dimension $n-1$. We now apply [2, Lemma 4] with $W=\mathbb{A}^{n}$, and $k_{i}=n-1$ for every index $i$. Taking $e \mid q$ with $e \geq A$ we find that there is a constant $C=C(d, n)$ such that

$$
N(e, A) \ll C^{\omega(e)}\left(A^{n} e^{-1}+\omega(e) A^{n-1}\right) \ll_{d, n, \eta} e^{\eta} A^{n-1}
$$

for any fixed $\eta>0$. It follows that if $e \geq A$ we will have

$$
\begin{aligned}
N(e, A) & =\frac{A^{n}}{e^{n}} N(e)+O\left(A^{n} N(e) e^{-n}\right)+O_{d, n, \eta}\left(A^{n-1} e^{\eta}\right) \\
& =\frac{A^{n}}{e^{n}} N(e)+O_{d, n, \eta}\left(A^{n} e^{-1+\eta}\right)+O_{d, n, \eta}\left(A^{n-1} e^{\eta}\right) \\
& =\frac{A^{n}}{e^{n}} N(e)+O_{d, n, \eta}\left(A^{n-1} e^{\eta}\right),
\end{aligned}
$$

so that (3.4) holds whether $e \leq A$ or not. 
We now examine

$$
\begin{aligned}
\# & \left\{\mathbf{a} \in \mathbb{N}^{n}: \max a_{i} \leq A,(F(\mathbf{a}), q)=1\right\} \\
& =\sum_{e \mid q} \mu(e) N(e, A) \\
& =\sum_{e \mid q} \mu(e) \frac{A^{n}}{e^{n}} N(e)+O_{d, n, \eta}\left(\sum_{e \mid q} A^{n-1} e^{\eta}\right) \\
& =A^{n} \prod_{p \mid q}\left(1-N(p) p^{-n}\right)+O_{d, n, \eta}\left(A^{n-1} q^{2 \eta}\right) .
\end{aligned}
$$

Since $N(p)<p^{n}$ and $N(p) \leq c_{0} p^{n-1}$ for some constant $c_{0}$ depending only on $d$ and $n$, we may deduce that

$$
\begin{aligned}
\prod_{p \mid q}\left(1-N(p) p^{-n}\right) & \geq \prod_{\substack{p \mid q \\
p \leq 2 c_{0}}}\left(1-\left(p^{n}-1\right) p^{-n}\right) \prod_{\substack{p \mid q \\
p>2 c_{0}}}\left(1-c_{0} p^{-1}\right) \\
& \geq \prod_{\substack{p \leq 2 c_{0} \\
p^{-n}}}\left(1-p^{-1}\right)^{2 c_{0}} \\
& \gg_{\substack{p \mid q \\
p>2 c_{0}}}\left(\frac{\phi(q)}{q}\right)^{2 c_{0}} \\
& \gg_{d, n, \eta} q^{-\eta} .
\end{aligned}
$$

It follows that

$$
\#\left\{\mathbf{a} \in \mathbb{N}^{n}: \max a_{i} \leq A,(F(\mathbf{a}), q)=1\right\} \geq c_{2} A^{n} q^{-\eta}-c_{3} A^{n-1} q^{2 \eta}
$$

for suitable positive constants $c_{2}$ and $c_{3}$ depending on $d$ and $n$. The lemma then follows on taking $\eta=\min (\varepsilon, \delta / 4)$.

\section{Proof of Theorem 3}

For the proof we will write

$$
\Sigma=\sum_{(x, y) \in C} \chi(Q(x, y))
$$

for convenience. Let $N \in \mathbb{N}$ be a parameter to be chosen, satisfying $N \leq$ $R q^{-1 / 100}$, say, and set $S=[R / N]$. We need to specify a "good" set of vectors $\underline{\mathrm{s}} \in \mathbb{N}^{2}$, and this will require a further definition. The form $Q(X, Y)$ should be thought of as lying in $(\mathbb{Z} / q \mathbb{Z})[X, Y]$, and we need an appropriate lift to $\mathbb{Z}[X, Y]$. To achieve this we write $Q\left(x_{1}, x_{2}\right)=A x_{1}^{2}+B x_{1} x_{2}+C x_{2}^{2}$ and

$$
\Lambda=\left\{\underline{\mathrm{v}} \in \mathbb{Z}^{3}: \underline{\mathrm{v}} \equiv \lambda(A, B, C)(\bmod q) \text { for some } \lambda \in \mathbb{Z}\right\},
$$

and we let $\left(A^{*}, B^{*}, C^{*}\right)$ be a non-zero vector in $\Lambda$ of minimal length. As there is a non-zero vector $\left(A^{\prime}, B^{\prime}, C^{\prime}\right) \equiv(A, B, C)(\bmod q)$ in $\Lambda$ with $\left|A^{\prime}\right|,\left|B^{\prime}\right|,\left|C^{\prime}\right| \leq q / 2$ we see that $q$ cannot divide $\left(A^{*}, B^{*}, C^{*}\right)$. We now define

$$
Q^{*}(X, Y)=A^{*} X^{2}+B^{*} X Y+C^{*} Y^{2} \text {. }
$$


Note that $\operatorname{det}\left(Q^{*}\right) \equiv \lambda^{2} \operatorname{det}(Q)(\bmod q)$ for an appropriate $\lambda$. Since $q$ cannot divide $\lambda$, and is square-free and coprime to $\operatorname{det}(Q)$, we will have $q \nmid \operatorname{det}\left(Q^{*}\right)$. In particular $Q^{*}$ is nonsingular, but there is no guarantee that $\left(\operatorname{det}\left(Q^{*}\right), q\right)=1$. We can now take our set of "good" vectors s to be

$$
\mathcal{S}=\left\{\left(s_{1}, s_{2}\right) \in \mathbb{N}^{2}:\|\mathbf{s}\| \leq S,(Q(\mathbf{s}), q)=1, Q^{*}(\underline{\mathbf{s}}) \neq 0\right\} .
$$

There are $O(S)$ vectors for which $Q^{*}(\underline{\mathrm{s}})=0$, uniformly over all non-zero forms $Q^{*}$. Thus, according to Lemma 5$]$ we have

$$
\# \mathcal{S} \gg_{\varepsilon} S^{2} q^{-\varepsilon}
$$

for $S \gg_{\varepsilon} q^{\varepsilon}$, for any fixed $\varepsilon>0$.

For any positive integer $n \leq N$ we proceed to write

$$
(\# \mathcal{S}) \Sigma=\sum_{\left(s_{1}, s_{2}\right) \in \mathcal{S}} \sum_{\left(x_{1}, x_{2}\right) \in Z^{2}} \chi\left(Q\left(x_{1}+n s_{1}, x_{2}+n s_{2}\right)\right) \mathbb{1}_{C}\left(x_{1}+n s_{1}, x_{2}+n s_{2}\right)
$$

where $\mathbb{1}_{C}$ is the characteristic function for the set $C$. It follows that

$$
N(\# \mathcal{S}) \Sigma=\sum_{\left(s_{1}, s_{2}\right) \in \mathcal{S}} \sum_{\left(x_{1}, x_{2}\right) \in Z^{2}} \sum_{n \in I} \chi\left(Q\left(x_{1}+n s_{1}, x_{2}+n s_{2}\right)\right),
$$

where

$$
I=\left\{n \leq N:\left(x_{1}+n s_{1}, x_{2}+n s_{2}\right) \in C\right\} .
$$

Since $C$ is convex, $I$ is an interval. Moreover if $I$ is nonempty, containing $\mathbf{x}+n \mathbf{s}$, then $\left\|\mathbf{x}+n \mathbf{s}-\mathbf{x}_{0}\right\| \leq R$ and $\|n \mathbf{s}\| \leq N S \leq R$, whence $\left\|\mathbf{x}-\mathbf{x}_{0}\right\| \leq 2 R$. We therefore deduce, via (4.2), that

$$
\Sigma \ll_{\varepsilon} N^{-1} S^{-2} q^{\varepsilon} \sum_{\mathbf{s} \in \mathcal{S}} \sum_{\substack{\mathbf{x} \in Z^{2} \\\left\|\mathbf{x}-\mathbf{x}_{0}\right\| \leq 2 R}} \max _{I \subseteq(0, N]}\left|\sum_{n \in I} \chi\left(Q\left(x_{1}+n s_{1}, x_{2}+n s_{2}\right)\right)\right| .
$$

If the reader compares this with the corresponding stage in the argument of Chang [7, see [7. (4.3)] for example, then it will be observed that Chang has a product $s t$ in place of our variable $n$. Indeed our method is slightly different from Chang's, requires one variable fewer, and does not use an argument corresponding to [7, Lemma 3].

To proceed further we use the readily verified identity

$$
Q\left(x_{1}+n s_{1}, x_{2}+n s_{2}\right)=Q(\mathbf{s}) \widetilde{Q}(n+a(\mathbf{s}, \mathbf{x}), b(\mathbf{s}, \mathbf{x})),
$$

where $\widetilde{Q}\left(x_{1}, x_{2}\right):=x_{1}^{2}+B x_{1} x_{2}+A C x_{2}^{2}$, and

$$
a(\mathbf{s}, \mathbf{x})=\frac{A x_{1} s_{1}+B x_{1} s_{2}+C x_{2} s_{2}}{Q\left(s_{1}, s_{2}\right)}, \quad b(\mathbf{s}, \mathbf{x})=\frac{x_{2} s_{1}-x_{1} s_{2}}{Q\left(s_{1}, s_{2}\right)} .
$$

Here the fractions are to be interpreted in the ring $\mathbb{Z} / q \mathbb{Z}$, the denominators $Q\left(s_{1}, s_{2}\right)$ being units by our choice of the set $\mathcal{S}$. We now write

$$
N(a, b)=\#\left\{(\mathbf{s}, \mathbf{x}) \in \mathcal{S} \times \mathbb{Z}^{2}:\left\|\mathbf{x}-\mathbf{x}_{0}\right\| \leq 2 R, a(\mathbf{s}, \mathbf{x})=a, b(\mathbf{s}, \mathbf{x})=b\right\},
$$


whence

$$
\Sigma \ll_{\varepsilon} N^{-1} S^{-2} q^{\varepsilon} \sum_{a, b(\bmod q)} N(a, b) \max _{I \subseteq(0, N]}\left|\sum_{n \in I} \chi(\widetilde{Q}(n+a, b))\right| .
$$

We must now consider the mean square of $N(a, b)$, for which we will prove the following bound.

Lemma 6 For any fixed $\varepsilon>0$ we have

$$
\sum_{a, b(\bmod q)} N(a, b)^{2} \ll_{\varepsilon} q^{\varepsilon} R^{2} S^{2}\left(1+R S q^{-1 / 2}+R^{2} S^{2} q^{-4 / 3}\right) .
$$

This will be established in the next section.

We also have the trivial bound

$$
\sum_{a, b(\bmod q)} N(a, b) \leq \#\left\{(\mathbf{s}, \mathbf{x}) \in \mathcal{S} \times \mathbb{Z}^{2}:\left\|\mathbf{x}-\mathbf{x}_{0}\right\| \leq 2 R\right\} \ll R^{2} S^{2},
$$

whence Hölder's inequality yields

$$
\begin{aligned}
& \Sigma^{2 r} \ll_{\varepsilon, r}\left(N^{-1} S^{-2} q^{\varepsilon}\right)^{2 r}\left\{\sum_{a, b(\bmod q)} N(a, b)\right\}^{2 r-2}\left\{\sum_{a, b(\bmod q)} N(a, b)^{2}\right\} \\
& \times \sum_{a, b(\bmod q)} \max _{I \subseteq(0, N]}\left|\sum_{n \in I} \chi(\widetilde{Q}(n+a, b))\right|^{2 r} \\
& \ll_{\varepsilon, r} \quad N^{-2 r} R^{4 r-2} S^{-2} q^{\varepsilon}\left(1+R S q^{-1 / 2}+R^{2} S^{2} q^{-4 / 3}\right) \\
& \times \sum_{a, b(\bmod q)} \max _{I \subseteq(0, N]}\left|\sum_{n \in I} \chi(\widetilde{Q}(n+a, b))\right|^{2 r} \\
& \ll_{\varepsilon, r} \quad N^{2-2 r} R^{4 r-4} q^{\varepsilon}\left(1+R^{2} N^{-1} q^{-1 / 2}+R^{4} N^{-2} q^{-4 / 3}\right) \\
& \times \sum_{a, b(\bmod q)} \max _{I \subseteq(0, N]}\left|\sum_{n \in I} \chi(\widetilde{Q}(n+a, b))\right|^{2 r},
\end{aligned}
$$

on employing our convention concerning the values taken by $\varepsilon$.

We are therefore led to consider sums of the form

$$
S(q ; H):=\sum_{a, b(\bmod q)}\left|\sum_{n \leq H} \chi(\widetilde{Q}(n+a, b))\right|^{2 r} .
$$

To estimate these we expand to obtain

$$
S(q ; H)=\sum_{n_{1}, \ldots, n_{2 r} \leq H} \Sigma(q ; \underline{\mathrm{n}})
$$

with

$$
\Sigma(q ; \underline{\mathrm{n}})=\sum_{a, b(\bmod q)} \chi\left(F_{+}(a, b ; \underline{\mathrm{n}})\right) \bar{\chi}\left(F_{-}(a, b ; \underline{\mathrm{n}})\right)
$$


and

$$
F_{+}(X, Y ; \underline{\mathrm{n}})=\prod_{i=1}^{r} \widetilde{Q}\left(n_{i}+X, Y\right), \quad F_{-}(X, Y ; \underline{\mathrm{n}})=\prod_{i=r+1}^{2 r} \widetilde{Q}\left(n_{i}+X, Y\right) .
$$

The sums $\Sigma(q ; \underline{\mathrm{n}})$ have a standard multiplicative property. If $q=u v$, say, then $u$ and $v$ will be coprime and square-free, and we can write $\chi=\chi_{u} \chi_{v}$ for suitable primitive characters to moduli $u$ and $v$ respectively. We will then have

$$
\Sigma(q ; \underline{\mathrm{n}})=\Sigma(u ; \underline{\mathrm{n}}) \Sigma(v ; \underline{\mathrm{n}}) .
$$

It therefore suffices to understand $\Sigma(q ; \underline{n})$ when $q$ is prime, for which we have the following result.

Lemma 7 Let $p$ be an odd prime not dividing $\operatorname{det}(\widetilde{Q})$, and let $\chi$ be a nonprincipal character to modulus $p$. Write

$$
\Delta_{i}=\prod_{\substack{1 \leq j \leq 2 r \\ j \neq i}}\left(n_{j}-n_{i}\right)
$$

and

$$
\Delta=\text { h.c.f. }\left(\Delta_{1}, \ldots, \Delta_{2 r}\right) \text {. }
$$

Then

$$
|\Sigma(p ; \underline{n})| \leq 4 r^{2} p(p, \Delta)
$$

We will prove this in Section [6. By summing over the $(2 r)$-tuples $\underline{n}$ we are then able to establish the following bound for $S(q ; H)$.

Lemma 8 For any $\varepsilon>0$ and $r \in \mathbb{N}$ we have

$$
S(q, H) \ll_{\varepsilon, r}(q H)^{\varepsilon}\left(q H^{2 r}+q^{2} H^{r}\right) .
$$

This will be proved in Section 7

Having established this there is a standard procedure to insert a maximum over subintervals of $(0, N]$, which goes back to Rademacher [15] and Menchov [14. We do not repeat the details, but instead refer the reader to Gallager and Montgomery [9, Section3] or Heath-Brown [13, Section 2]. The outcome is the following result.

Lemma 9 For any $\varepsilon>0$ and $r \in \mathbb{N}$ we have

$$
\sum_{a, b(\bmod q)} \max _{I \subseteq(0, N]}\left|\sum_{n \in I} \chi(\widetilde{Q}(n+a, b))\right|^{2 r} \ll_{\varepsilon, r}(q N)^{\varepsilon}\left(q N^{2 r}+q^{2} N^{r}\right) .
$$

We are now ready to complete the proof of Theorem 3. We insert the bound of Lemma 9 into (4.4), to give

$\Sigma^{2 r} \ll_{\varepsilon, r} N^{2-2 r} R^{4 r-4} q^{\varepsilon}\left(1+R^{2} N^{-1} q^{-1 / 2}+R^{4} N^{-2} q^{-4 / 3}\right) \cdot(q N)^{\varepsilon}\left(q N^{2 r}+q^{2} N^{r}\right)$.

In order to balance the final two terms we choose $N=\left[q^{1 / r}\right]$, which satisfies our constraint $N \leq R q^{-1 / 100}$ provided that $R \geq q^{1 / 4+1 / 2 r}$ and $r \geq 3$. On re-defining $\varepsilon$ we then find that

$$
\begin{array}{rll}
\Sigma^{2 r} & \ll_{\varepsilon, r} & q^{\varepsilon} N^{2-2 r} R^{4 r-4}\left(1+R^{2} N^{-1} q^{-1 / 2}+R^{4} N^{-2} q^{-4 / 3}\right) \cdot q N^{2 r} \\
& \ll_{\varepsilon, r} & q^{1 / 2+1 / r+\varepsilon} R^{4 r-2}\left(R^{-2} q^{1 / 2+1 / r}+1+R^{2} q^{-5 / 6-1 / r}\right),
\end{array}
$$

and the theorem follows. 


\section{Proof of Lemma 6}

We now prove Lemma 6. In view of the definitions (4.3) we have the identity

$$
(A b(\mathbf{s}, \mathbf{x}) X-a(\mathbf{s}, \mathbf{x}) Y)\left(s_{2} X-s_{1} Y\right)=s_{2} b(\mathbf{s}, \mathbf{x}) Q(X, Y)-\left(x_{2} X-x_{1} Y\right) Y
$$

in $(\mathbb{Z} / q \mathbb{Z})[X, Y]$. Thus if $a(\mathbf{s}, \mathbf{x})=a\left(\mathbf{s}^{\prime}, \mathbf{x}^{\prime}\right)=a$ and $b(\mathbf{s}, \mathbf{x})=b\left(\mathbf{s}^{\prime}, \mathbf{x}^{\prime}\right)=b$ then

$$
\begin{aligned}
(A b X-a Y)\left(s_{2} X-s_{1} Y\right)\left(s_{2}^{\prime} X-s_{1}^{\prime} Y\right) & \\
= & \left(s_{2} b Q(X, Y)-\left(x_{2} X-x_{1} Y\right) Y\right)\left(s_{2}^{\prime} X-s_{1}^{\prime} Y\right),
\end{aligned}
$$

and also

$$
\begin{aligned}
(A b X-a Y)\left(s_{2}^{\prime} X-s_{1}^{\prime} Y\right)\left(s_{2} X-s_{1} Y\right) & \\
= & \left(s_{2}^{\prime} b Q(X, Y)-\left(x_{2}^{\prime} X-x_{1}^{\prime} Y\right) Y\right)\left(s_{2} X-s_{1} Y\right) .
\end{aligned}
$$

Thus by subtraction we deduce that

$$
\begin{array}{r}
Y\left\{\left(x_{2} X-x_{1} Y\right)\left(s_{2}^{\prime} X-s_{1}^{\prime} Y\right)-\left(x_{2}^{\prime} X-x_{1}^{\prime} Y\right)\left(s_{2} X-s_{1} Y\right)\right\} \\
=b\left(s_{2}^{\prime} s_{1}-s_{1}^{\prime} s_{2}\right) Y Q(X, Y),
\end{array}
$$

still in $(\mathbb{Z} / q \mathbb{Z})[X, Y]$.

We then deduce that

$$
\left(x_{2} s_{2}^{\prime}-x_{2}^{\prime} s_{2}, x_{2}^{\prime} s_{1}+x_{1}^{\prime} s_{2}-x_{2} s_{1}^{\prime}-x_{1} s_{2}^{\prime}, x_{1} s_{1}^{\prime}-x_{1}^{\prime} s_{1}\right) \in \Lambda,
$$

with $\Lambda$ given by (4.1). It follows that

$$
\sum_{a, b} N(a, b)^{2} \leq \sum_{\mathbf{s}, \mathbf{s}^{\prime} \in \mathcal{S}} N_{1}\left(\mathbf{s}, \mathbf{s}^{\prime}\right)
$$

where $N_{1}\left(\mathbf{s}, \mathbf{s}^{\prime}\right)$ counts pairs of vectors $\left(\mathbf{x}, \mathbf{x}^{\prime}\right)$ each lying in the disc $\left\|\mathbf{x}-\mathbf{x}_{0}\right\| \leq$ $2 R$, such that (5.1) holds. Now suppose that $\left(\mathbf{x}_{1}, \mathbf{x}_{1}^{\prime}\right)$ is a pair counted by $N_{1}\left(\mathbf{s}, \mathbf{s}^{\prime}\right)$. For any other such pair we write $\mathbf{x}=\mathbf{x}_{1}+\underline{u}$ and $\mathbf{x}^{\prime}=\mathbf{x}_{1}^{\prime}+\underline{u}^{\prime}$ whence, by subtraction, we find firstly that $\|\underline{u}\|, \| \underline{u}^{\prime}|| \leq 4 R$, and secondly that

$$
\left(u_{2} s_{2}^{\prime}-u_{2}^{\prime} s_{2}, u_{2}^{\prime} s_{1}+u_{1}^{\prime} s_{2}-u_{2} s_{1}^{\prime}-u_{1} s_{2}^{\prime}, u_{1} s_{1}^{\prime}-u_{1}^{\prime} s_{1}\right) \in \Lambda .
$$

Thus $N_{1}\left(\mathbf{s}, \mathbf{s}^{\prime}\right) \leq N_{2}\left(\mathbf{s}, \mathbf{s}^{\prime}\right)$, where $N_{2}\left(\mathbf{s}, \mathbf{s}^{\prime}\right)$ counts pairs of vectors $\underline{u}, \underline{u}^{\prime}$ satisfying (5.2), and having length at most $4 R$.

We have already chosen $\left(A^{*}, B^{*}, C^{*}\right)=\underline{\mathrm{v}}_{1}$, say, as the shortest vector in $\Lambda$. As in the proof of Davenport [8, Lemma5], we can then construct a basis $\underline{\mathrm{v}}_{1}, \underline{\mathrm{v}}_{2}, \underline{\mathrm{v}}_{3}$ for $\Lambda$, such that if $\underline{\mathrm{v}}=\lambda_{1} \underline{\mathrm{v}}_{1}+\lambda_{2} \underline{\mathrm{v}}_{2}+\lambda_{3} \underline{\mathrm{v}}_{3}$, then $\lambda_{i} \ll\left\|\underline{\mathrm{v}}|| /|| \underline{\mathrm{v}}_{i}\right\|$ for $i=1,2,3$. Moreover we will have

$$
\left\|\underline{\mathrm{v}}_{1}\right\| \leq\left\|\underline{\mathrm{v}}_{2}\right\| \leq\left\|\underline{\mathrm{v}}_{3}\right\|
$$

and

$$
\left\|\underline{\mathrm{v}}_{1}\right\| \cdot \cdot\left|\underline{\mathrm{v}}_{2}\left\|\cdot \mid \underline{\mathrm{w}}_{3}\right\| \geq \operatorname{det}(\Lambda) .\right.
$$

In our case we have $\operatorname{det}(\Lambda)=q^{2}$, whence

$$
\left\|\underline{\mathrm{v}}_{2}\right\| .\left\|\underline{\mathrm{v}}_{3}\right\| \geq q^{4 / 3} .
$$


Moreover one sees from the definition of $\Lambda$ that $q \mid \underline{\mathrm{v}}_{1} \wedge \underline{\mathrm{v}}_{2}$, and since the vectors $\underline{\mathrm{v}}_{1}$ and $\underline{\mathrm{v}}_{2}$ are not proportional it follows that

$$
q \leq\left\|\underline{\mathrm{v}}_{1} \wedge \underline{\mathrm{v}}_{2}\right\| \leq\left\|\underline{\mathrm{v}}_{1}\right\| \cdot\left\|\underline{\mathrm{v}}_{2}\right\| \leq\left\|\underline{\mathrm{v}}_{2}\right\|^{2} .
$$

The vector

$$
\underline{\mathrm{v}}=\left(u_{2} s_{2}^{\prime}-u_{2}^{\prime} s_{2}, u_{2}^{\prime} s_{1}+u_{1}^{\prime} s_{2}-u_{2} s_{1}^{\prime}-u_{1} s_{2}^{\prime}, u_{1} s_{1}^{\prime}-u_{1}^{\prime} s_{1}\right)
$$

has length at most $32 R S$ so that the corresponding coefficients satisfy

$$
\lambda_{2} \ll \frac{R S}{\left\|\underline{\mathrm{v}}_{2}\right\|} \text { and } \lambda_{3} \ll \frac{R S}{\left\|\underline{\mathrm{v}}_{3}\right\|} .
$$

If we break the available vectors counted by $N_{2}\left(\mathbf{s}, \mathbf{s}^{\prime}\right)$ into subsets according to the values of $\lambda_{2}$ and $\lambda_{3}$, then the number of such subsets will be

$$
\ll\left(1+\frac{R S}{\left\|\underline{\mathrm{v}}_{2}\right\|}\right)\left(1+\frac{R S}{\left\|\underline{\mathrm{v}}_{3}\right\|}\right) \ll 1+\frac{R S}{\left\|\underline{\mathrm{v}}_{2}\right\|}+\frac{R^{2} S^{2}}{\left\|\underline{\mathrm{v}}_{2}\right\| \cdot\left\|\underline{\mathrm{v}}_{3}\right\|} \ll 1+\frac{R S}{q^{1 / 2}}+\frac{R^{2} S^{2}}{q^{4 / 3}} .
$$

If $\left(\underline{\mathrm{u}}_{1}, \underline{\mathrm{u}}_{1}^{\prime}\right)$ and $\left(\underline{\mathrm{u}}_{2}, \underline{\mathrm{u}}_{2}^{\prime}\right)$ are two pairs belonging to the same subset, and we write $\underline{\mathrm{u}}=\underline{\mathrm{u}}_{1}-\underline{\mathrm{u}}_{2}$ and $\underline{\mathrm{u}}^{\prime}=\underline{\mathrm{u}}_{1}^{\prime}-\underline{\mathrm{u}}_{2}^{\prime}$, then

$$
\underline{\mathrm{v}}=\left(u_{2} s_{2}^{\prime}-u_{2}^{\prime} s_{2}, u_{2}^{\prime} s_{1}+u_{1}^{\prime} s_{2}-u_{2} s_{1}^{\prime}-u_{1} s_{2}^{\prime}, u_{1} s_{1}^{\prime}-u_{1}^{\prime} s_{1}\right)
$$

will be a multiple of $\underline{\mathrm{v}}_{1}=\left(A^{*}, B^{*}, C^{*}\right)$, and we will have $\|\underline{\mathrm{u}}\|,\left\|\underline{\mathrm{u}}^{\prime}\right\| \leq 8 R$.

We therefore conclude that

$$
N_{2}\left(\mathbf{s}, \mathbf{s}^{\prime}\right) \ll\left(1+R S q^{-1 / 2}+R^{2} S^{2} q^{-4 / 3}\right) N_{3}\left(\mathbf{s}, \mathbf{s}^{\prime}\right)
$$

where $N_{3}\left(\mathbf{s}, \mathbf{s}^{\prime}\right)$ counts pairs of vectors $\underline{u}, \underline{u}^{\prime}$ having length at most $8 R$, and for which the vector (5.3) is an integer multiple of $\left(A^{*}, B^{*}, C^{*}\right)$. The quadratic form corresponding to $\underline{\mathrm{v}}$ is

$$
\begin{aligned}
& \left(u_{2} s_{2}^{\prime}-u_{2}^{\prime} s_{2}\right) X^{2}+\left(u_{2}^{\prime} s_{1}+u_{1}^{\prime} s_{2}-u_{2} s_{1}^{\prime}-u_{1} s_{2}^{\prime}\right) X Y+\left(u_{1} s_{1}^{\prime}-u_{1}^{\prime} s_{1}\right) Y^{2} \\
& \quad=\left(u_{2} X-u_{1} Y\right)\left(s_{2}^{\prime} X-s_{1}^{\prime} Y\right)-\left(u_{2}^{\prime} X-u_{1}^{\prime} Y\right)\left(s_{2} X-s_{1} Y\right) .
\end{aligned}
$$

We therefore conclude that

$$
Q^{*}(X, Y) \mid\left(u_{2} X-u_{1} Y\right)\left(s_{2}^{\prime} X-s_{1}^{\prime} Y\right)-\left(u_{2}^{\prime} X-u_{1}^{\prime} Y\right)\left(s_{2} X-s_{1} Y\right) .
$$

Thus, to complete the proof of Lemma 6 it suffices to show that

$$
\begin{gathered}
\#\left\{\left(\underline{\mathrm{u}}, \underline{\mathrm{u}}^{\prime}, \underline{\mathrm{s}}, \underline{\mathrm{s}}^{\prime}\right) \in \mathbb{Z}^{2} \times \mathbb{Z}^{2} \times \mathcal{S} \times \mathcal{S}:\|\underline{\mathrm{u}}\|,\left\|\underline{\mathrm{u}}^{\prime}\right\| \leq 8 R,(\underline{5.4}) \text { holds }\right\} \\
\ll_{\varepsilon} q^{\varepsilon} R^{2} S^{2} .
\end{gathered}
$$

Given two binary quadratic forms $Q_{1}$ and $Q_{2}$ one may define a covariant $C\left(Q_{1}, Q_{2}\right)$ as the discriminant of the binary form $D(\alpha, \beta)=\operatorname{det}\left(\alpha Q_{1}+\beta Q_{2}\right)$. One readily confirms that $C\left(Q_{1}, Q_{2}\right)=C\left(Q_{1}+\lambda Q_{2}, Q_{2}\right)$ for any constant $\lambda$, and moreover that

$$
C\left(\left(u_{2} X-u_{1} Y\right)\left(v_{2} X-v_{1} Y\right), Q\right)=Q\left(u_{1}, u_{2}\right) Q\left(v_{1}, v_{2}\right) .
$$


Taking $Q_{1}=\left(u_{2} X-u_{1} Y\right)\left(s_{2}^{\prime} X-s_{1}^{\prime} Y\right)$ and $Q_{2}=Q^{*}$ we deduce that

$$
Q^{*}\left(u_{1}, u_{2}\right) Q^{*}\left(s_{1}^{\prime}, s_{2}^{\prime}\right)=Q^{*}\left(u_{1}^{\prime}, u_{2}^{\prime}\right) Q^{*}\left(s_{1}, s_{2}\right) .
$$

In defining the set $\mathcal{S}$ we arranged that $Q^{*}(\underline{\mathrm{s}}) \neq 0$. If $Q^{*}(\underline{\mathrm{u}}) \neq 0$ then $Q^{*}\left(u_{1}, u_{2}\right) Q^{*}\left(s_{1}^{\prime}, s_{2}^{\prime}\right)$ has $O_{\varepsilon}\left(q^{\varepsilon}\right)$ divisors, since

$$
\left.\left.\left|Q^{*}\left(u_{1}, u_{2}\right) Q^{*}\left(s_{1}^{\prime}, s_{2}^{\prime}\right)\right| \ll \max \left(\left|A^{*}\right|,\left|B^{*}\right|,\left|C^{*}\right|\right)^{2}|| \underline{\mathbf{u}}\right|^{2}|| \underline{\mathrm{s}}\right|^{2} \ll q^{2} R^{2} S^{2} \ll q^{6} .
$$

Moreover, when $d \neq 0$ the equation $Q^{*}\left(u_{1}^{\prime}, u_{2}^{\prime}\right)=d$ will have $\ll_{\varepsilon}(q R)^{\varepsilon} \ll_{\varepsilon} q^{\varepsilon}$ solutions $\underline{\mathrm{u}}^{\prime}$ with $\left\|\underline{\mathrm{u}}^{\prime}\right\| \leq 8 R$, by Theorem 13 of Heath-Brown [12] for example. (Here we use crucially the fact that $Q^{*}$ is nonsingular.) Similarly $Q^{*}\left(s_{1}, s_{2}\right)=d^{\prime}$ will have $O_{\varepsilon}\left(q^{\varepsilon}\right)$ solutions for any $d^{\prime} \neq 0$. It then follows that the contribution arising from 4-tuples $\left(\underline{\mathrm{u}}, \underline{\mathrm{u}}^{\prime}, \underline{\mathrm{s}}, \underline{\mathrm{s}}^{\prime}\right)$ in which $Q^{*}(\underline{\mathrm{u}}) \neq 0$ will be $O_{\varepsilon}\left(q^{\varepsilon} R^{2} S^{2}\right)$, which is satisfactory for (5.5).

It remains to deal with the case in which $Q^{*}(\underline{\underline{u}})=0$. In view of (5.6) we will then have $Q^{*}\left(\underline{\underline{u}}^{\prime}\right)=0$, since $Q^{*}(\underline{\mathrm{s}})$ and $Q^{*}\left(\underline{\mathrm{s}}^{\prime}\right)$ are non-zero. We now claim that either $\underline{\mathrm{u}}=\underline{\mathrm{u}}^{\prime}=\underline{0}$, or $Q^{*}(X, Y)$ factors over $\mathbb{Z}$ into linear factors $L_{1}(X, Y)$ and $L_{2}(X, Y)$ such that $L_{1}(X, Y)$ divides both $u_{2} X-u_{1} Y$ and $u_{2}^{\prime} X-u_{1}^{\prime} Y$. To see this, suppose that $\underline{u} \neq \underline{0}$, say. Then we must have $L_{1}(X, Y) \mid u_{2} X-u_{1} Y$ for some integral linear factor of $Q^{*}$. It would then follow from (5.4) that $L_{1}(X, Y) \mid u_{2}^{\prime} X-u_{1}^{\prime} Y$, since $Q^{*}(\underline{\mathrm{s}}) \neq 0$. The claim then follows.

Clearly the contribution to (5.5) arising from the case $\underline{u}=\underline{u}^{\prime}=\underline{0}$ is $O\left(S^{4}\right)=$ $O\left(R^{2} S^{2}\right)$, which is satisfactory, so it remains to consider the case in which

$$
u_{2} X-u_{1} Y=k L_{1}(X, Y) \text { and } u_{2}^{\prime} X-u_{1}^{\prime} Y=k^{\prime} L_{1}(X, Y)
$$

with integers $k, k^{\prime}$ such that $|k|,\left|k^{\prime}\right| \leq 8 R$. We then have

$$
k\left(s_{2}^{\prime} X-s_{1}^{\prime} Y\right) \equiv k^{\prime}\left(s_{2} X-s_{1} Y\right)\left(\bmod L_{2}(X, Y)\right),
$$

by (5.4). If $L_{2}(X, Y)=a X-b Y$, say then we must have

$$
k\left(s_{2}^{\prime} b-s_{1}^{\prime} a\right)=k^{\prime}\left(s_{2} b-s_{1} a\right) .
$$

Moreover $s_{2}^{\prime} b-s_{1}^{\prime} a$ and $s_{2} b-s_{1} a$ are non-zero, since $Q^{*}(\underline{\mathrm{s}})$ and $Q^{*}\left(\underline{\mathrm{s}}^{\prime}\right)$ do not vanish. If $k^{\prime}=0$ then $k=0$, which would put us in the case $\underline{u}=\underline{u}^{\prime}=\underline{0}$ which has already been dealt with. Since at most one of $a$ or $b$ can vanish we may suppose that $b$ say, is non-zero. There are then $O\left(R S^{3}\right)$ possibilities for $s_{1}^{\prime}, s_{1}, s_{2}$ and $k^{\prime}$, and the number of divisors $k$ of $k^{\prime}\left(s_{2} b-s_{1} a\right)$ will be $O_{\varepsilon}\left((q S)^{\varepsilon}\right)$, since $|a|,|b| \ll \max \left(\left|A^{*}\right|,\left|B^{*}\right|,\left|C^{*}\right|\right) \ll q$. The complementary divisor to $k$ is then $s_{2}^{\prime} b-s_{1}^{\prime} a$, which determines $s_{2}^{\prime}$. We therefore conclude that the corresponding contribution to (5.5) is $O_{\varepsilon}\left(q^{\varepsilon} R^{2} S^{2}\right)$, since $S \leq R \leq q$. This completes the proof of (5.5), and hence of Lemma 6 .

\section{Proof of Lemma 7}

Our proof of Lemma 7 is inspired by the viewpoint taken by Chang 7 . We first consider the case in which $\widetilde{Q}(X, Y)=X^{2}+B X Y+A C Y^{2}$ factors modulo $p$. In this case we may replace $\widetilde{Q}(X, Y)$ by $(X+\lambda Y)(X+\mu Y)$ say, where $p \nmid \lambda-\mu$ since $p \nmid \operatorname{det}(\widetilde{Q})$. Then $\widetilde{Q}(n+a, b)=\left(n+a^{\prime}\right)\left(n+b^{\prime}\right)$ where $a^{\prime}=a+\lambda b$ and 
$b^{\prime}=a+\mu b$ are independent of $n$. Moreover $\left(a^{\prime}, b^{\prime}\right)$ runs over $\mathbb{F}_{p}^{2}$ as $(a, b)$ does. It follows that

$$
\Sigma(p ; \underline{\underline{n}})=\sum_{a, b(\bmod p)} \chi\left(G_{+}(a, b ; \underline{\underline{n}})\right) \bar{\chi}\left(G_{-}(a, b ; \underline{\underline{n}})\right)
$$

with

$$
G_{+}(X, Y ; \underline{\mathrm{n}})=\prod_{i=1}^{r}\left(n_{i}+X\right)\left(n_{i}+Y\right), \quad G_{-}(X, Y ; \underline{\mathrm{n}})=\prod_{i=r+1}^{2 r}\left(n_{i}+X\right)\left(n_{i}+Y\right) .
$$

We then see that

$$
\Sigma(p ; \underline{\underline{n}})=\Sigma_{1}(p ; \underline{\underline{n}})^{2}
$$

with

$$
\Sigma_{1}(p ; \underline{\mathrm{n}})=\sum_{a(\bmod p)} \chi\left(H_{+}(a ; \underline{\mathrm{n}})\right) \bar{\chi}\left(H_{-}(a ; \underline{\mathrm{n}})\right)
$$

and

$$
H_{+}(X ; \underline{\mathrm{n}})=\prod_{i=1}^{r}\left(n_{i}+X\right), \quad H_{-}(X ; \underline{\mathrm{n}})=\prod_{i=r+1}^{2 r}\left(n_{i}+X\right) .
$$

The sum $\Sigma_{1}(p ; \underline{\underline{n}})$ occurs in the work of Burgess [3, Lemma 1], from which one readily sees that

$$
\left|\Sigma_{1}(p ; \underline{\mathrm{n}})\right| \leq 2 r \sqrt{p}
$$

unless every linear factor of the polynomial $H_{+}(X ; \underline{\underline{n}}) H_{-}(X ; \underline{\underline{n}})$ has multiplicity two or more, modulo $p$. In the exceptional case we have $p \mid \Delta_{i}$ for every $i$, whence $p \mid \Delta$. We deduce that (6.1) holds whenever $p \nmid \Delta$. In the remaining case we have a trivial bound $\left|\Sigma_{1}(p ; \underline{n})\right| \leq p$, so that

$$
\left|\Sigma_{1}(p ; \underline{\underline{n}})\right| \leq 2 r p^{1 / 2}(p, \Delta)^{1 / 2}
$$

whether or not $p \nmid \Delta$. We therefore conclude that

$$
|\Sigma(p ; \underline{\mathrm{n}})| \leq 4 r^{2} p(p, \Delta)
$$

whenever $\widetilde{Q}$ factors modulo $p$. This is satisfactory for Lemma 7 .

We turn now to the case in which $\widetilde{Q}$ is irreducible over $\mathbb{F}_{p}$. It will be typographically convenient to write $F$ for the field $\mathbb{F}_{p^{2}}$. In the case under consideration, there is a factorization $\widetilde{Q}(X, Y)=(X+\lambda Y)\left(X+\lambda^{\prime} Y\right)$ say, over $F$ with $\lambda$ and $\lambda^{\prime}$ being conjugates in $F / \mathbb{F}_{p}$. We may now define a function $\psi$ from $F$ to $\mathbb{C}$ by setting

$$
\psi(a+\lambda b)=\chi\left((a+\lambda b)\left(a+\lambda^{\prime} b\right)\right)=\chi(\widetilde{Q}(a, b)) .
$$

One easily sees that this is a non-trivial multiplicative character on $F$, and that

$$
\Sigma(p ; \underline{\mathrm{n}})=\sum_{\alpha \in F} \psi\left(H_{+}(\alpha ; \underline{\mathrm{n}})\right) \bar{\psi}\left(H_{-}(\alpha ; \underline{\mathrm{n}})\right) .
$$

Burgess' proof of (6.1), based on Weil's "Riemann Hypothesis" for curves over arbitrary finite fields, immediately extends to $\Sigma(p ; \underline{\mathrm{n}})$, and shows that

$$
|\Sigma(p ; \underline{\mathrm{n}})| \leq 2 r \sqrt{\# F}=2 r p
$$


unless every linear factor of the polynomial $H_{+}(X ; \underline{\underline{n}}) H_{-}(X ; \underline{\underline{n}})$ has multiplicity two or more, modulo $p$. In the alternative case we have $p \mid \Delta$, in the notation of the lemma, and we deduce that

$$
|\Sigma(p ; \underline{\mathrm{n}})| \leq p(p, \Delta),
$$

in view of the trivial bound $|\Sigma(p ; \underline{n})| \leq p^{2}$. As above, these bounds are satisfactory for Lemma 7

\section{Proof of Lemma 8}

It follows from Lemma 7, along with the multiplicative relation (4.5) that

$$
\Sigma(q ; \underline{\underline{n}}) \leq\left(4 r^{2}\right)^{\omega(q)} q(q, \Delta) \ll_{\varepsilon, r} q^{1+\varepsilon}(q, \Delta) .
$$

Thus to prove Lemma 8 it will be enough to show that

$$
\sum_{n_{1}, \ldots, n_{2 r} \leq H}(q, \Delta) \ll_{\varepsilon, r}(q H)^{\varepsilon}\left(H^{2 r}+q H^{r}\right) .
$$

Indeed, we have

$$
\sum_{n_{1}, \ldots, n_{2 r} \leq H}(q, \Delta) \leq \sum_{k \mid q} k \#\left\{\underline{\underline{n}} \in \mathbb{N}^{2 r} \cap(0, H]^{2 r}: k \mid \Delta\right\},
$$

so that it suffices to establish the estimate

$$
\#\left\{\underline{\mathrm{n}} \in \mathbb{N}^{2 r} \cap(0, H]^{2 r}: k \mid \Delta\right\} \ll_{\varepsilon, r}(k H)^{\varepsilon}\left(H^{2 r} k^{-1}+H^{r}\right) .
$$

We first consider vectors $\underline{n}$ for $\Delta_{1}=\ldots=\Delta_{2 r}=0$. Then if $\nu \in \mathbb{N}$ and there is any index $i$ such that $n_{i}=\nu$, there must be at least two such indices. It follows that the set $\left\{n_{1}, \ldots, n_{2 r}\right\}$ contains at most $r$ distinct elements. There are at most $H^{r}$ choices for these elements, $\nu_{1}, \ldots, \nu_{s}$ say, with $1 \leq s \leq r$. Once the $\nu_{j}$ have been chosen there are (at most) $s$ choices for each $n_{i}$. It follows that there are $O_{r}\left(H^{r}\right)$ vectors $\underline{n}$ for which $\Delta_{1}=\ldots=\Delta_{2 r}=0$. This is satisfactory for (7.1).

In the remaining case we have $\Delta_{j} \neq 0$ for some index $j$, and

$$
\begin{aligned}
\#\left\{\underline{\mathrm{n}} \in \mathbb{N}^{2 r} \cap(0, H]^{2 r}: k \mid \Delta, \Delta \neq 0\right\} \\
\quad \leq \sum_{j=1}^{2 r} \#\left\{\underline{\mathrm{n}} \in \mathbb{N}^{2 r} \cap(0, H]^{2 r}: k \mid \Delta_{j}, \Delta_{j} \neq 0\right\} .
\end{aligned}
$$

However $\left|\Delta_{j}\right| \leq H^{2 r-1}$, so that there are at most $2 H^{2 r-1} k^{-1}$ possibilities for $\Delta_{j}$. For each such choice of $\Delta_{j}$ there are at most $2 d\left(\left|\Delta_{j}\right|\right) \ll_{\varepsilon, r} H^{\varepsilon}$ possibilities for each of its divisors $n_{i}-n_{j}$. Thus, taking account of the $O(H)$ possibilities for $n_{j}$ itself, we find that

$$
\#\left\{\underline{\underline{\mathrm{n}}} \in \mathbb{N}^{2 r} \cap(0, H]^{2 r}: k \mid \Delta_{j}, \Delta_{j} \neq 0\right\} \ll_{\varepsilon, r} H^{2 r-1} k^{-1}\left(H^{\varepsilon}\right)^{2 r-1} H .
$$

After replacing $\varepsilon$ by $\varepsilon /(2 r-1)$ we see that this is $O_{\varepsilon, r}\left(H^{2 r+\varepsilon} k^{-1}\right)$. Since this is satisfactory for (7.1) the proof of Lemma 8 is complete. 


\section{Acknowledgement}

This research was supported by EPSRC grant EP/K021132X/1.

\section{References}

[1] R.C. Baker, Diophantine inequalities (London Math. Soc. Monographs New Series, 1, Oxford, 1986).

[2] T.D. Browning and D.R. Heath-Brown, Rational points on quartic hypersurfaces, J. Reine Angew. Math., 629 (2009), 37-88.

[3] D.A. Burgess, On character sums and primitive roots, Proc. London Math. Soc. (3), 12 (1962), 179-192.

[4] D.A. Burgess, On character sums and L-series. II, Proc. London Math. Soc. (3), 13 (1963), 524-536.

[5] D.A. Burgess, On the quadratic character of a polynomial, J. London Math. Soc., 42 (1967), 73-80.

[6] D.A. Burgess, A note on character sums of binary quadratic forms, $J$. London Math. Soc., 43 (1968), 271-274.

[7] M.-C. Chang, Burgess inequality in $\mathbb{F}_{p^{2}}$, Geom. Funct. Anal., 19 (2009), 1001-1016.

[8] H. Davenport, Cubic forms in sixteen variables, Proc. Roy. Soc. Ser. A, 272 (1963), 285-303.

[9] P.X. Gallagher and H.L. Montgomery, On the Burgess estimate, Math. Notes 88 (2010), 321-329.

[10] D.R. Heath-Brown, Small solutions of quadratic congruences, Glasgow Math. J., 27 (1985), 87-93.

[11] D.R. Heath-Brown, Small solutions of quadratic congruences, II, Mathematika, 38 (1991), no. 2, 264-284 (1992).

[12] D.R. Heath-Brown, The density of rational points on cubic surfaces, Acta Arith., 79 (1997), 17-30.

[13] D.R. Heath-Brown, Burgess's bounds for character sums, Number theory and related fields, 199-213, Springer Proc. Math. Stat., 43, (Springer, New York, 2013).

[14] D. Menchov, Sur les séries de fonctions orthogonales, Fund. Math., 1 (1923), $82-105$.

[15] H. Rademacher, Einige Sätze über Reihen von allgemeinen OrthogonalFunktionen, Math. Ann., 87 (1922), 112-138

[16] A. Schinzel, H.-P. Schlickewei, and W.M. Schmidt, Small solutions of quadratic congruences and small fractional parts of quadratic forms, Acta Arith., 37 (1980), 241-248. 
Mathematical Institute,

Radcliffe Observatory Quarter,

Woodstock Road,

Oxford

OX2 6GG

UK

rhb@maths.ox.ac.uk 